\title{
Dust grain dynamics due to nonuniform and nonstationary high-frequency radiations in cold magnetoplasmas
}

\author{
A. K. Nekrasov and F. Z. Feygin \\ Institute of Physics of the Earth, Russian Academy of Sciences, 123995 Moscow, Russia \\ Received: 16 August 2004 - Revised: 10 January 2006 - Accepted: 16 January 2006 - Published: 23 March 2006
}

\begin{abstract}
A general nonlinear theory for low-frequency electromagnetic field generation due to high-frequency nonuniform and nonstationary electromagnetic radiations in cold, uniform, multicomponent, dusty magnetoplasmas is developed. This theory permits us to consider the nonlinear action of all waves that can exist in such plasmas. The equations are derived for the dust grain velocities in the lowfrequency nonlinear electric fields arising due to the presence of electromagnetic cyclotron waves travelling along the background magnetic field. The dust grains are considered to be magnetized as well as unmagnetized. Different regimes for the dust particle dynamics, depending on the spatio-temporal change of the wave amplitudes and plasma parameters, are discussed. It is shown that induced nonlinear electric fields can have both an electrostatic and electromagnetic nature. Conditions for maximum dust acceleration are found. The results obtained may be useful for understanding the possible mechanisms of dust grain dynamics in astrophysical, cosmic and laboratory plasmas under the action of nonuniform and nonstationary electromagnetic waves.
\end{abstract}

Keywords. Electromagnetics (Electromagnetic theory; Nonlinear electromagnetic; Plasmas)

\section{Introduction}

Dusty plasma physics is a rapidly growing field. The presence of charged dust grains is ubiquitous in various lowtemperature plasmas, in the Earth's lower ionosphere and in astrophysical objects (Shukla et al., 1995). The first detection of dust particles in the Earth's mesosphere (height 80 to $100 \mathrm{~km}$ ) by direct rocket probe measurements was reported a few years ago (Havnes et al., 1996). Interstellar dust streams penetrate into our solar system (Altobelli et al., 2003). The spatial distribution of dust grains can be nonuniform, for example, comets possess long dust tails. Dust in planetary magnetospheres is concentrated in rings, where some rings

Correspondence to: A. K. Nekrasov

(anatoli.nekrassov@t-online.de) have radial and azimuthal optical depth modulations (Goertz, 1989).

Dusty plasmas are characterized as an ensemble of micron-sized, extremely massive, charged dust grains, usually immersed in a fully or partially ionized low-temperature electron-ion plasma. Dust grains can be one billion times heavier than protons and have charges up to several thousand times that of the electron charge. Dust grains are charged both negatively, due to absorption of electrons from the background plasma (Goertz, 1989; Barkan et al., 1994), and positively, due to various processes, such as photoionization, thermoionic emission induced by radiative heating, and secondary electron emission (Fortov et al., 1998). For example, in the Earth's mesosphere (Havnes et al., 1996) the charge state can be 20 , the dust radius $0.5 \mu \mathrm{m}$, the ratio of the dust to proton mass $2 \times 10^{8}$, and the ratio of the dust number density to that of the ions can be 0.05 .

The dynamics of dusty plasmas can considerably differ from that of an ordinary electron-ion plasma. The dust particles can change the properties of the plasma itself and also change the dispersion relations of various waves (Shukla, 1995; Verheest, 2000). The physics of collective processes in dusty plasmas has attracted much attention, following the discovery of dust acoustic waves (Rao et al., 1990) and dust plasma crystals (Chu and Lin, 1994; Thomas et al., 1994). The theoretical idea for the latter was put forward by Ikezi (1986). When the Coulomb interaction between charged grains is much smaller than their thermal energy, dust grains may, as usual, be treated as a gas of very heavy, multiply charged, negative and/or positive ions. In the opposite case dust plasma crystals can be formed.

The dust grain dynamic influences on the cosmic microwave background (Ferrara and Dettmar, 1994; Draine and Lazarian, 1998). This is a consequence of the interaction of dust with background electron-ion plasma and electromagnetic fields. One of the important questions of dust grain dynamics is the movement of charged grains as a result of the action of the high-frequency radiation existing in the environment. High-frequency waves can produce ponderomotive forces leading to mass transport and particle energization. There are many works dealing with these processes in

Published by Copernicus GmbH on behalf of the European Geosciences Union. 
the laboratory (e.g. Fisch and Boozer, 1980; Lee and Parks, 1983; Abe and Kadoya, 1988; Myra and D'Ippolito, 2000), magnetospheric (Li and Temerin, 1993; Allan and Manuel, 1996, and references therein; Shukla et al., 1996; Guglielmi and Lundin, 2001; Nekrasov and Feygin, 2005) and solar (Ofman and Davila, 1995; Fränz et al., 2000) electron-ion plasmas. The investigations have shown that the ponderomotive force can considerably modify the plasma state and accelerate the particles. It is obvious that analogous processes must also occur in dusty plasmas. The accelerated dust winds and dust fluxes are observed in the cosmic environment and late-type stars (Spitzer Jr., 1978; Falceta-Goncalves and Jatenco-Pereira, 2002; Altobelli et al., 2003). Experiments are carried out on the interaction of radar beams with the mesospheric dusty plasma (Havnes et al., 1992, 2001). The ponderomotive force can be used, in particular, for removal of impurities (dust grains) from the plasma in laboratory devices. Thus, the study of the dust grain dynamics in high-frequency electromagnetic fields is important for both practical purposes and for the explanation of natural phenomena.

The dust grain acceleration due to ponderomotive force of electromagnetic cyclotron, lower-hybrid and Alfvén waves has been examined (Shukla et al., 2002; Shukla and Stenflo, 2002; Shukla and Schlickeiser, 2003). Dust grains have been shown to be subjected to the action of the low-frequency, nonlinear electric field induced by the ponderomotive force. The high-frequency wave amplitudes have been considered to depend mainly on the space coordinates. However, in nature waves exist, as a rule, in nonuniform, nonstationary and/or unstable media, and therefore their amplitudes change dynamically. The nonlinear effects define the dependence of large-wave amplitudes on space and time. In experiments the external radiation, for example, a laser pulse, also has spatiotemporal variations (e.g. Jha et al., 2004; Liu et al., 2004). In a general case the relationship between the spatial and temporal changes of the wave amplitude may be various. It results in different dust grain dynamics, which are considered here.

In the present paper we develop a general nonlinear theory for low-frequency electromagnetic field generation under the action of high-frequency electromagnetic radiations in cold, multicomponent, dusty magnetoplasmas. This theory will permit us to consider the nonlinear influence of all waves that can exist in such plasmas. As an example for high-frequency waves we use electron and ion cyclotron waves travelling along the background magnetic field. We derive the equations for the dust grain velocities arising due to the nonlinear electric fields. We discuss various cases of dust grain movements. The dust grains are considered to be magnetized as well as unmagnetized. We find conditions for the stationary and dynamic regimes, which depend on the spatio-temporal changes in the high-frequency wave amplitude.

In Sect. 2 we derive the general equation for nonlinear electric fields in cold, multicomponent magnetoplasmas.
Then, we deduce the solution of this equation for the case when the high-frequency waves are circularly polarized electromagnetic waves travelling along the background magnetic field. In Sect. 3 we obtain the equations for the velocities of magnetized and unmagnetized dust grains, which do not take part in fast oscillations. The stationary and dynamic regimes are considered here. The equation for the dust number density is given. For all cases considered the conditions for neglecting the thermal pressure are presented. In Sect. 4 we discuss our results and their possible geophysical applications. In Sect. 5 the results obtained are summarized.

\section{Basic equations and expressions}

\subsection{General relations}

We start with the equations of motion, continuity and Maxwell's equations

$$
\begin{aligned}
& \frac{\partial \mathbf{v}_{j}}{\partial t}+\mathbf{v}_{j} \cdot \nabla \mathbf{v}_{j}=\frac{q_{j}}{m_{j}} \mathbf{E}+\frac{q_{j}}{m_{j} c} \mathbf{v}_{j} \times\left(\mathbf{B}_{0}+\mathbf{B}\right), \\
& \frac{\partial n_{j}}{\partial t}+\nabla \cdot n_{j} \mathbf{v}_{j}=0, \\
& \nabla \times \mathbf{E}=-\frac{1}{c} \frac{\partial \mathbf{B}}{\partial t}, \\
& \nabla \times \mathbf{B}=\frac{4 \pi}{c} \mathbf{j}+\frac{1}{c} \frac{\partial \mathbf{E}}{\partial t} .
\end{aligned}
$$

Here $\mathbf{j}=\sum_{j} q_{j} n_{j} \mathbf{v}_{j}, q_{j}$ and $m_{j}$ are the charge and mass of species $j, \mathbf{v}_{j}$ is the hydrodynamic velocity, $n_{j}$ is the number density, $\mathbf{E}$ and $\mathbf{B}$ are the perturbed electric and magnetic fields, $\mathbf{B}_{0}$ is the background magnetic field, and $c$ is the speed of light in a vacuum. Here we do not take into account the thermal pressure. The necessary conditions for that omission will be given below.

Next, we are going to obtain the equations for the nonoscillatory part of $\mathbf{v}_{j}$. For this purpose we consider, at first, Eqs. (1-4) to the first order in the wave amplitude. The background magnetic field $\mathbf{B}_{0}$ is supposed to be uniform and directed along the $\mathrm{z}$-axis, and the background number density $n_{j 0}$ is also uniform. For simplicity, all variables are considered to depend only on the coordinates $y$ and $z$. Then, we first rederive in the linear approximation the well-known Fourier expressions for the velocity and number density perturbations

$$
\begin{aligned}
\mathbf{v}_{j 1 \perp k} & =\frac{q_{j}}{m_{j}} \frac{i \omega \mathbf{E}_{1 \perp k}-\omega_{c j} \mathbf{E}_{1 k} \times \mathbf{b}_{0}}{\omega^{2}-\omega_{c j}^{2}}, \\
v_{j 1 z k} & =\frac{q_{j}}{m_{j}} \frac{i}{\omega} E_{1 z k}
\end{aligned}
$$

and

$n_{j 1 k}=n_{j 0} \frac{\mathbf{k} \cdot \mathbf{v}_{j 1 k}}{\omega}$, 
where $\omega_{c j}=q_{j} B_{0} / m_{j} c$ is the cyclotron frequency, $\mathbf{b}_{0}=\mathbf{B}_{0} / B_{0}$, index "1" denotes the linear approximation, index " $\perp$ " marks the direction across $\mathbf{B}_{0}, k=\{\mathbf{k}, \omega\}$, and $\mathbf{k}$ is the wave vector and $\omega$ is the frequency. Using Eqs. (5), we thus deduce the linear current $\mathbf{j}_{1 k}$, assuming that the background velocity is $\mathbf{v}_{j 0}=\mathbf{0}$. Substituting this current into the linearized versions of the Maxwell Eqs. (3) and (4), we obtain in the Fourier representation the ordinary equations for the wave electric field components (see, for example, Akhiezer et al., 1974)

$\hat{\mathbf{A}}_{k} \mathbf{E}_{1 k}=0$,

where the matrix $\hat{\mathbf{A}}_{k}$ is

$\hat{\mathbf{A}}_{k}=\left|\begin{array}{ccc}n^{2}-\varepsilon_{1}, & -i \varepsilon_{2}, & 0 \\ i \varepsilon_{2}, & n_{z}^{2}-\varepsilon_{1}, & -n_{y} n_{z} \\ 0, & -n_{y} n_{z}, & n_{y}^{2}-\varepsilon_{3}\end{array}\right|$.

Here $n^{2}=n_{y}^{2}+n_{z}^{2}, n_{y}=k_{y} c / \omega, n_{z}=k_{z} c / \omega$, whereas $\varepsilon_{1}, \varepsilon_{2}$ and $\varepsilon_{3}$ are the components of the dielectric permeability tensor, i.e.

$\varepsilon_{1}=1-\sum_{j} \frac{\omega_{p j}^{2}}{\omega^{2}-\omega_{c j}^{2}}$,

$\varepsilon_{2}=-\sum_{j} \frac{\omega_{p j}^{2} \omega_{c j}}{\left(\omega^{2}-\omega_{c j}^{2}\right) \omega}$,

$\varepsilon_{3}=1-\sum_{j} \frac{\omega_{p j}^{2}}{\omega^{2}}$,

where $\omega_{p j}=\left(4 \pi n_{j 0} q_{j}^{2} / m_{j}\right)^{1 / 2}$ is the plasma frequency.

Let us now consider the equation of motion (1) up to the second order in the wave amplitude, introducing the nonlinear force

$\mathbf{F}_{j 2}=-\frac{m_{j}}{q_{j}} \mathbf{v}_{j 1} \cdot \nabla \mathbf{v}_{j 1}+\frac{1}{c} \mathbf{v}_{j 1} \times \mathbf{B}_{1}$.

Note that the ponderomotive force is $\left\langle\mathbf{F}_{j 2}\right\rangle$, where (and below) the angle brackets $\langle\ldots\rangle$ denote time-averaging over fast oscillations. Index "2" denotes here and below the quantities of the second order. Then, for the velocity $\mathbf{v}_{j 2}$ we obtain the same equation as for $\mathbf{v}_{j 1}$, if we make the substitutions: "1" $\rightarrow$ "2", and $\mathbf{E}_{1} \rightarrow \mathbf{E}_{2}+\mathbf{F}_{j 2}$. Therefore, the solution for $\mathbf{v}_{j 2 k}$ has a form which is similar to Eqs. (5). Substituting the nonlinear currents into Maxwell's equations, we then obtain the equation for the nonlinear electric field $\mathbf{E}_{2 k}$

$\hat{\mathbf{A}}_{k} \mathbf{E}_{2 k}=\mathbf{C}_{k}$,

where

$\mathbf{C}_{k}=\frac{4 \pi i}{\omega} \sum_{j} q_{j} n_{j 1} \mathbf{v}_{j 1}-\mathbf{D}_{k}$, with

$\mathbf{D}_{\perp k}=\sum_{j} \frac{\omega_{p j}^{2}}{\omega^{2}-\omega_{c j}^{2}}\left(\mathbf{F}_{j 2 \perp k}+i \frac{\omega_{c j}}{\omega} \mathbf{F}_{j 2 k} \times \mathbf{b}_{0}\right)$

and

$D_{z k}=\sum_{j} \frac{\omega_{p j}^{2}}{\omega^{2}} F_{j 2 z k}$.

All expressions obtained above have been written in a general form and are suitable for various waves, which can exist in cold, uniform, multicomponent magnetoplasmas. Note that by using Eqs. (3) and (11), we can also find the induced magnetic moment of medium in various cases, for example, those considered below.

\subsection{Circularly polarized waves}

Below, as an example, we will focus our attention on circularly polarized electromagnetic waves travelling along the background magnetic field $\mathbf{B}_{0}$, i.e. $n_{z}^{2} \gg n_{y}^{2}$ and $\varepsilon_{3} \gg n_{y}^{2}$. These waves are used, in particular, for investigation of the mesospheric dusty plasma (Havnes et al., 1992, 2001). In this case the dispersion relation has the form

$n_{z}^{2}=\varepsilon_{1}+\sigma \frac{\omega}{|\omega|} \varepsilon_{2}$,

and the relationship between field components is

$E_{1 x k}=i \sigma \frac{\omega}{|\omega|} E_{1 y k}$,

where $\sigma= \pm 1$, and the sign $\|$ denotes the absolute value. When $\sigma=+1(-1)$ the wave is left- (right-) polarized. The contribution of the particle oscillations along the background magnetic field to the dispersion relation (14) can obviously be neglected.

Let us consider plasma motions varying slowly with respect to the wave frequencies. For this purpose we average Eq. (11) over fast oscillations, supposing that the wave amplitudes depend on the coordinates $y, z$ and time $t$, and that the wave phases do not depend on the coordinate $y$. We then obtain from Eq. (11):

$\varepsilon\left\langle E_{2 x}\right\rangle_{k}=\left(n_{z}^{2}-\varepsilon_{1}\right)\left\langle C_{x}\right\rangle_{k}+i \varepsilon_{2}\left(\left\langle C_{y}\right\rangle_{k}-\frac{n_{y} n_{z}}{\varepsilon_{3}}\left\langle C_{z}\right\rangle_{k}\right)$,
$\varepsilon\left\langle E_{2 y}\right\rangle_{k}=-i \varepsilon_{2}\left\langle C_{x}\right\rangle_{k}+\left(n^{2}-\varepsilon_{1}\right)\left(\left\langle C_{y}\right\rangle_{k}-\frac{n_{y} n_{z}}{\varepsilon_{3}}\left\langle C_{z}\right\rangle_{k}\right)$,
$\varepsilon_{3}\left\langle E_{2 z}\right\rangle_{k}=-\left\langle C_{z}\right\rangle_{k}$,

where

$\varepsilon=\left(n^{2}-\varepsilon_{1}\right)\left(n_{z}^{2}-\varepsilon_{1}\right)-\varepsilon_{2}^{2}$.

The wave vector $\mathbf{k}$ and frequency $\omega$ in Eqs. (16) characterize the inhomogeneity and nonstationarity of the wave amplitude. For the components of the nonlinear "driving force" $\langle\mathbf{C}\rangle_{k}$ we obtain the expressions 


$$
\begin{aligned}
\left\langle C_{x}\right\rangle_{k} & =\frac{k_{y}}{\omega} \sum_{j} \frac{q_{j}}{2 m_{j} \omega_{c j}} \frac{\omega_{p j}^{2}}{\left(\sigma \omega^{\prime}-\omega_{c j}\right)^{2}}\left\langle\mathbf{E}_{1}^{2}\right\rangle_{k}, \\
\left\langle C_{y}\right\rangle_{k} & =-i k_{y} \sum_{j} \frac{q_{j}}{4 m_{j} \omega_{c j}^{2}} \frac{\omega_{p j}^{2}}{\omega^{\prime 2}}\left[\frac{\sigma \omega^{\prime}\left(2 \sigma \omega^{\prime}-\omega_{c j}\right)}{\left(\sigma \omega^{\prime}-\omega_{c j}\right)^{2}}+\left(1+\frac{\omega_{c j}}{\sigma \omega^{\prime}-\omega_{c j}} \frac{k_{z} \omega^{\prime}}{\omega k_{z}^{\prime}}\right) \frac{\omega_{c j}}{\sigma \omega^{\prime}} \frac{n_{z}^{\prime 2}}{\varepsilon_{3}^{\prime}}\right]\left\langle\mathbf{E}_{1}^{2}\right\rangle_{k}, \\
\left\langle C_{z}\right\rangle_{k} & =\frac{i}{\omega^{2}} \sum_{j} \frac{q_{j}}{2 m_{j}} \frac{\omega_{p j}^{2}}{\sigma \omega^{\prime}\left(\sigma \omega^{\prime}-\omega_{c j}\right)}\left(k_{z}+\frac{\omega_{c j}}{\sigma \omega^{\prime}-\omega_{c j}} \frac{k_{z}^{\prime}}{\omega^{\prime}} \omega\right)\left\langle\mathbf{E}_{1}^{2}\right\rangle_{k} .
\end{aligned}
$$

Here and below the index "'" denotes the wave number and frequency of the linear wave. The frequency $\omega^{\prime}$ is assumed to be fixed. The wave number $k_{z}^{\prime}$ is determined from Eq. (14). When calculating Eqs. (17) the relationship (15) has been used. We suppose that $\omega^{2} \ll \omega_{c j}^{2}$ for particle species that participate in the fast oscillations.

We see from Eqs. (16) that the magnitude of the nonlinear electric field $\left\langle\mathbf{E}_{2 \perp}\right\rangle$ depends, to a large extent, on the quantity $\varepsilon_{2}$ (see Eqs. 9). When all particle species are magnetized with respect to $\omega\left(\omega^{2} \ll \omega_{c j}^{2}\right)$, then $\varepsilon_{2}$ is very small $\left(\varepsilon_{1} \gg \varepsilon_{2} \sim \omega\right)$. However, if, for example, the dust grains are unmagnetized, then $\varepsilon_{2}$ can be large $\left(\varepsilon_{2} \sim \omega^{-1}\right)$. Therefore, we consider these two cases separately.

In our analysis here we do not take into account the thermal pressure of plasma. The necessary condition for such an approximation for fast oscillations in the case $T_{\perp j} \sim T_{|| j}$ has the form $1 \gg\left(T_{|| j} / m_{j}\right) k_{z}^{\prime 2} / \omega^{\prime 2}$, where $T_{\perp(||) j}$ is the temperature of the species $j, j=e, i$ represents electrons and ions, respectively, and indices " $\perp$ " and "I" mark directions relatively to $\mathbf{B}_{0}$. We suppose that the dust grains do not participate in fast oscillations, i.e. $\omega^{\prime 2} \gg \omega_{c d}^{2}, \omega_{p d}^{2}$, where $d$ denotes the dust grains. The conditions for neglecting the thermal motion for slow movements will be given below.

\section{Nonlinear dynamics of dust grains}

\subsection{Magnetized dust grains}

In this section we consider the case when the dust grains are magnetized, i.e. $\omega^{2} \ll \omega_{c d}^{2}$. This inequality means that the amplitude of the high-frequency field changes sufficiently slowly, and/or the background magnetic field is sufficiently large. In this case the value $\varepsilon_{2}$, taking into account the equilibrium quasi-neutrality condition $n_{e 0}=n_{i 0}\left(1+\delta_{d}\right)$, where $\delta_{d}=q_{d} n_{d 0} / e n_{i 0}\left(-q_{e}=q_{i}=e\right)$, is much smaller than $\varepsilon_{1}$ and has the form $\varepsilon_{2}=\sum_{j} \omega_{p j}^{2} \omega / \omega_{c j}^{3}$, whereas $\varepsilon_{1}$ is $\varepsilon_{1}=c^{2} / c_{A}^{2}$, where $c_{A}=B_{0} /\left[4 \pi\left(\sum_{j} n_{j 0} m_{j}\right)\right]^{1 / 2}$ is the Alfvén velocity $\left(\varepsilon_{1} \gg 1\right)$. Further, as can be seen from Eqs. (16), we can consider two various cases in the dependence on the relation between $n_{z}^{2}$ and $\varepsilon_{1}$.

\subsubsection{Stationary regime}

We now suppose that $\omega^{2} \ll k_{z}^{2} c_{A}^{2}$. In this case $\left\langle E_{2 x}\right\rangle_{k}=\left\langle C_{x}\right\rangle_{k} / n^{2}$ and $\left\langle E_{2 y}\right\rangle_{k}=-i \varepsilon_{2}\left\langle C_{x}\right\rangle_{k} / n^{2} n_{z}^{2}-\left(k_{y} / k_{z}\right)$ $\left\langle C_{z}\right\rangle_{k} / \varepsilon_{3}$. In the expression for $\left\langle C_{z}\right\rangle_{k}$ the second (nonstationary) term in the brackets (see Eqs. 17) can be neglected.

Let us now present the equations for the velocity $\left\langle\mathbf{v}_{d 2}\right\rangle$ of the dust grains. In the expressions (5), written for the values of the second order $(1 \rightarrow 2)$, we must then use the electric field $\left\langle\mathbf{E}_{2}\right\rangle$. As a result, from Eqs. (17) and at $\varepsilon_{3} \gg 1$ we find

$$
\begin{aligned}
& \left\langle v_{d 2 x}\right\rangle_{k}=\sum_{j \neq d} \frac{q_{j}}{2 m_{j}} \frac{\omega_{p j}^{2}}{\omega_{p e}^{2}\left(\sigma \omega^{\prime}-\omega_{c j}\right)^{2}}\left[\frac{\sigma \omega^{\prime}-\omega_{c j}}{\sigma \omega^{\prime}}-\frac{\omega_{p e}^{2}}{\omega_{c d} \omega_{c j} n^{2}}\right] \frac{c}{B_{0}} i k_{y}\left\langle\mathbf{E}_{1}^{2}\right\rangle_{k}, \\
& \left\langle v_{d 2 y}\right\rangle_{k}=\sum_{j \neq d} \frac{q_{j}}{2 m_{j}} \frac{\omega_{p j}^{2}}{\omega_{p e}^{2}\left(\sigma \omega^{\prime}-\omega_{c j}\right)^{2}}\left[\frac{\sigma \omega^{\prime}-\omega_{c j}}{\sigma \omega^{\prime}}-\frac{\omega_{p e}^{2} \omega_{c d}}{\omega_{c j} \omega^{2} n^{2}}\right] \frac{c}{B_{0}} \frac{\omega}{\omega_{c d}} k_{y}\left\langle\mathbf{E}_{1}^{2}\right\rangle_{k}, \\
& \frac{\partial\left\langle v_{d 2 z}\right\rangle}{\partial t}=-\frac{q_{d} e}{2 m_{d} m_{e}} \frac{1}{\sigma \omega^{\prime}\left(\sigma \omega^{\prime}-\omega_{c e}\right)}\left[\frac{\partial}{\partial z}-\frac{\omega_{c e}}{\sigma \omega^{\prime}-\omega_{c e}} \frac{k_{z}^{\prime}}{\omega^{\prime}} \frac{\partial}{\partial t}\right]\left\langle\mathbf{E}_{1}^{2}\right\rangle .
\end{aligned}
$$


The equation for $\left\langle v_{d 2 z}\right\rangle$ is given in a general form. In the stationary regime $k_{z} / \omega \gg k_{z}^{\prime} / \omega^{\prime}$.

When the second term in the square brackets in the expression for $\left\langle v_{d 2 y}\right\rangle_{k}$ is larger than the first term in the square brackets in the expression for $\left\langle v_{d 2 x}\right\rangle_{k}$, i.e. $k^{2} c_{A i}^{2} \ll \omega \omega_{c i}$ (with $\omega^{\prime} \sim \omega_{c j}$ for this inequality), where $k^{2}=k_{y}^{2}+k_{z}^{2}$ and $c_{A i}=B_{0} /\left(4 \pi n_{i 0} m_{i}\right)^{1 / 2}$ is the ion Alfvén velocity, we have from Eqs. (18): $\left\langle v_{d 2 y}\right\rangle=-\left(c / B_{0}\right)\left\langle E_{2 x}\right\rangle,\left\langle v_{d 2 y}\right\rangle \gg\left\langle v_{d 2 x}\right\rangle$ and

$$
\left(\frac{\partial^{2}}{\partial y^{2}}+\frac{\partial^{2}}{\partial z^{2}}\right) v_{d 2 y}=\frac{1}{2 B_{0}^{2}}\left[\sum_{j \neq d} \frac{\omega_{p j}^{2}}{\left(\sigma \omega^{\prime}-\omega_{c j}\right)^{2}}\right] \frac{\partial^{2}\left\langle\mathbf{E}_{1}^{2}\right\rangle}{\partial y \partial t} \text {. }
$$

Note that in this regime the electric field $\left\langle\mathbf{E}_{2}\right\rangle$ has an electromagnetic nature. And finally, for the purely stationary regime, when $k^{2} c_{A i}^{2} \gg \omega \omega_{c i}$, we obtain

$$
\left\langle v_{d 2 x}\right\rangle=\frac{c}{B_{0}}\left\langle E_{2 y}\right\rangle=-\frac{e}{2 m_{e}} \frac{1}{\sigma \omega^{\prime}\left(\sigma \omega^{\prime}-\omega_{c e}\right)} \frac{c}{B_{0}} \frac{\partial\left\langle\mathbf{E}_{1}^{2}\right\rangle}{\partial y},
$$

with $\left\langle v_{d 2 y}\right\rangle \ll\left\langle v_{d 2 x}\right\rangle$. Here the electric field $\left\langle E_{2 y}\right\rangle$ is related to $\left\langle E_{2 z}\right\rangle$, i.e. $\partial\left\langle E_{2 y}\right\rangle / \partial z=\partial\left\langle E_{2 z}\right\rangle / \partial y$. Thus, the induced electric field has an electrostatic nature in the purely stationary regime. Note that the field $\left\langle E_{2 z}\right\rangle$ is only determined by the electron motion (see Eqs. 16 and 17). We see that the transverse field $\left\langle\mathbf{E}_{2 \perp}\right\rangle$ has different directions in the two considered cases $k^{2} c_{A i}^{2} \ll(\gg) \omega \omega_{c i}$.

The relationship between transverse and longitudinal velocities depends on the concrete spatio-temporal inhomogeneity of the high-frequency wave amplitude.

The equation for the dust grain number density evolution here and below is

$$
\frac{\partial\left\langle n_{d 2}\right\rangle}{\partial t}=-n_{d 0}\left(\frac{\partial\left\langle v_{d 2 y}\right\rangle}{\partial y}+\frac{\partial\left\langle v_{d 2 z}\right\rangle}{\partial z}\right) .
$$

The conditions for neglecting the thermal pressure in the slow movements are $1 \gg\left(T_{\perp j} / m_{j}\right) k_{y}^{2} / \omega_{c j}^{2}$; $\left(T_{|| j} / m_{j}\right) k_{z}^{2} / \omega^{2} ; c_{s}^{2} / c_{A i}^{2}$ and $1 \gg \alpha_{j}\left(T_{\perp e} / m_{e}\right) k_{z}^{2} / \omega^{2}$, where $c_{s}=\left[\left(\sum_{j} n_{j 0} T_{\perp j}\right) / n_{i 0} m_{i}\right]^{1 / 2}$ is the ion sound speed, $\alpha_{j} \sim \omega_{c j} / \omega^{\prime} \quad\left(\omega^{\prime}<\right.$ or $\sim \omega_{c j}, \omega^{\prime}$ is the frequency of corresponding waves).

\subsubsection{Dynamic regime}

Suppose that $\omega^{2} \gg k^{2} c_{A}^{2}$. In this case we obtain from Eqs. (16): $\left\langle E_{2 x}\right\rangle=-\left\langle C_{x}\right\rangle / \varepsilon_{1}$ and $\left\langle E_{2 y}\right\rangle \ll\left\langle E_{2 x}\right\rangle$. Using Eqs. (5), $(1 \rightarrow 2)$, we find the equation for the transverse dust grain velocity

$$
\frac{\partial\left\langle v_{d 2 y}\right\rangle}{\partial t}=-\frac{c_{A}^{2}}{2 B_{0}^{2}}\left[\sum_{j \neq d} \frac{\omega_{p j}^{2}}{\left(\sigma \omega^{\prime}-\omega_{c j}\right)^{2}}\right] \frac{\partial\left\langle\mathbf{E}_{1}^{2}\right\rangle}{\partial y} .
$$

In the dynamic regime the equations for accelerations along and across the background magnetic field can have the same form if $\omega^{\prime} / k_{z}^{\prime} \gg c_{A}$. In this case it is possible that $k_{z} / \omega \gg k_{z}^{\prime} / \omega^{\prime}$ in the equation for $\left\langle v_{d 2 z}\right\rangle$ (see Eqs. 18). Then we have $(j=e, i)$

$$
\frac{\left\langle v_{d 2 z}\right\rangle}{\left\langle v_{d 2 y}\right\rangle} \sim \frac{\omega_{c d}}{\omega_{c i}} \frac{c_{A i}^{2}}{c_{A}^{2}} \frac{k_{z}}{k_{y}} \frac{\omega_{c j}}{\omega^{\prime}} .
$$

Comparing these velocities we see that the transverse velocity of the dust grains in the dynamic regime, when $n_{i 0} m_{i} \gg n_{d 0} m_{d}(j=e)$, is, roughly, $\omega_{c i} / \omega_{c d} \gg 1$ times larger than the longitudinal one. In the opposite case, when $n_{i 0} m_{i} \ll n_{d 0} m_{d}$, this relation depends on the value $\delta_{d}$, and stays the same if $\delta_{d} \ll 1$. We note that the particle inertia in the slow movements containing in $\varepsilon_{1}$ must be taken into account for the dynamic regime.

The conditions for neglecting the thermal motion in the dynamic regime are similar to those above, except for the last inequality, which now is $1 \gg \alpha_{j}\left(T_{\perp e} / m_{e}\right) k_{z} k_{z}^{\prime} / \omega \omega^{\prime}$.

\subsection{Unmagnetized dust grains}

Here we suppose that $\omega^{2} \gg \omega_{c d}^{2}$. Then

$\varepsilon_{1} \simeq \frac{\omega_{p i}^{2}}{\omega_{c i}^{2}}-\frac{\omega_{p d}^{2}}{\omega^{2}}$,

$\varepsilon_{2}=-\delta_{d} \frac{\omega_{p i}^{2}}{\omega \omega_{c i}}$.

(for simplicity, we consider $m_{i} \gg \delta_{d} m_{e}$ ). The second term on the right-hand side in the equality for $\varepsilon_{1}$ is less than $\varepsilon_{2}$ by a factor of $\omega / \omega_{c d} \gg 1$.

If $\varepsilon \gg \varepsilon_{2}^{2}$, we return to the cases considered in Sect. 3.1, but now with $\varepsilon_{1} \simeq c^{2} / c_{A i}^{2}$. In the stationary regime, $n_{z}^{2} \gg \varepsilon_{1}$, the frequency $\omega$ must be much smaller than $k_{z} c_{A i}$ and $k k_{z} c_{A i}^{2} / \delta_{d} \omega_{c i}$. In the dynamic regime, $n^{2} \ll \varepsilon_{1}$, we have $\omega \gg k c_{A i}$ and $\delta_{d} \omega_{c i}$. This regime is possible for $\delta_{d} \ll 1$, as far as $\omega \ll \omega_{c i}$. In Eq. (21) for $\left\langle v_{d 2 y}\right\rangle$ the Alfvén velocity $c_{A}$ must be replaced by $c_{A i}$.

Let us now look at $\varepsilon_{2} \gg n^{2}-\varepsilon_{1}$ or $\delta_{d} \omega_{c i} \gg \omega \gg k^{2} c_{A i}^{2} / \delta_{d} \omega_{c i}$. This regime can be considered as a quasi-dynamic one. In this case the inequalities $n_{d 0} m_{d} \gg n_{i 0} m_{i}$ (as far as $\omega \gg \omega_{c d}$ ) and $\delta_{d} \gg k c_{A i} / \omega_{c i}$ must be fulfilled. Then, we obtain from Eqs. (16) $\left\langle E_{2 y}\right\rangle_{k}=i\left\langle C_{x}\right\rangle_{k} / \varepsilon_{2}$ and $\left\langle E_{2 x}\right\rangle_{k} \ll\left\langle E_{2 y}\right\rangle_{k}$. We thus find

$$
\frac{\partial\left\langle v_{2 d y}\right\rangle}{\partial t}=-\frac{1}{8 \pi n_{d 0} m_{d}}\left[\sum_{j \neq d} \frac{\omega_{p j}^{2}}{\left(\sigma \omega^{\prime}-\omega_{c j}\right)^{2}}\right] \frac{\partial\left\langle\mathbf{E}_{1}^{2}\right\rangle}{\partial y} .
$$

The equation for $\left\langle v_{2 d z}\right\rangle$ remains the same as above. It is followed that the field $\left\langle\mathbf{E}_{2}\right\rangle$ is the electromagnetic one.

We see from Eqs. (21) and (22) that for $n_{d 0} m_{d} \gg n_{i 0} m_{i}$ the transverse acceleration of magnetized dust grains in the dynamic regime coincides with the acceleration of unmagnetized grains, when $\delta_{d} \omega_{c i} \gg \omega$ (quasi-dynamic regime). In the opposite case, $\delta_{d} \omega_{c i} \ll \omega$ (dynamic regime), the acceleration of unmagnetized grains is $n_{d 0} m_{d} / n_{i 0} m_{i} \gg 1$ times larger. 
When $n_{d 0} m_{d} \ll n_{i 0} m_{i}$, the accelerations of magnetized and unmagnetized grains coincide in the dynamic regimes.

The thermal pressure in the quasi-dynamic regime can be neglected if $1 \gg T_{\perp d} k_{y}^{2} / m_{d} \omega^{2}$ and $\delta_{d}^{2} \gg k_{y}^{2} c_{s i}^{2} / \omega_{c i}^{2}$, where $c_{s i}^{2}=\left(n_{e 0} T_{\perp e}+n_{i 0} T_{\perp i}\right) / n_{i 0} m_{i}$, and if the same conditions as those for magnetized grains in the stationary and dynamic regimes are fulfilled (except $1 \gg T_{\perp d} k_{y}^{2} / m_{d} \omega_{c d}^{2}$ ).

3.3 Velocity expressions for electron and ion cyclotron waves

Finally, we present the velocity expressions in the cases of electromagnetic electron and ion cyclotron waves. From Eq. (14) we obtain the dispersion relation for electron cyclotron waves $\left(\sigma=-1, \omega^{\prime} \gg \omega_{c i}\right)$

$n_{z}^{\prime 2}=1+\frac{\omega_{p e}^{2}}{\omega^{\prime}\left(\left|\omega_{c e}\right|-\omega^{\prime}\right)}$.

In the equations for the velocities it is necessary to put $\sigma=-1$, and in Eqs. (21) and (22) to take $j=e, i$.

In the case of ion cyclotron waves the dispersion relation is $\left(\omega^{\prime} \ll\left|\omega_{c e}\right|\right)$

$n_{z}^{\prime 2}=1+\frac{\omega_{p i}^{2}}{\omega_{c i}\left(\omega_{c i}-\sigma \omega^{\prime}\right)}\left(1-\delta_{d} \frac{\omega_{c i}-\sigma \omega^{\prime}}{\sigma \omega^{\prime}}\right)$

where $\sigma= \pm 1$. In the equations for velocities we must put $\omega^{\prime} \ll\left|\omega_{c e}\right|$ and in Eqs. (21) and (22) take $j=i$, because the contribution of the electrons is very small (when $m_{i} \gg \delta_{d} m_{e}$ ).

\section{Discussion - possible geophysical applications}

The main goal of the present investigation is the consideration of the dust grain dynamics due to the spatio-temporal inhomogeneity of the ponderomotive force. Usually, the ponderomotive force is considered to be stationary, and only the spatial gradients of the high-frequency wave amplitude are taken into account. In this case one should suppose that waves do not suffer linear and nonlinear instabilities and maintain their regular shape (e.g. Shukla and Schlickeiser, 2003). In other words, the processes must be stationary. But it is clear that these conditions are far from always being fulfilled in real situations, such as magnetospheric storms, Sun flares, generation of impulsive radiations, plasma instabilities, nonstationary nonlinear processes and turbulence, and so on. Accordingly, the rise of charged particle fluxes, in particular, dust winds, has a dynamical character.

Two consequences are followed from our consideration: 1) depending on the spatio-temporal inhomogeneity of the high-frequency wave amplitude the transverse dust grain velocity can be directed both along (Eqs. 19, 21,22) and across (Eq. 20) the amplitude inhomogeneity; 2) the induced nonlinear electric field driving the dust grains has mainly an electromagnetic nature (this field is electrostatic only in the purely stationary regime, see Eq. 20) and can be directed both across (cases 19,21) and along (cases 20, 22) the wave amplitude inhomogeneity.

One more important issue of our calculations concerns with the longitudinal electron and ion accelerations under the action of the circularly polarized waves. It can be shown that $\left(1+\delta_{d}\right) \partial\left\langle v_{e 2 z}\right\rangle / \partial t=\partial\left\langle v_{i 2 z}\right\rangle / \partial t=-\left(e / m_{i}\right)\left(\left\langle F_{e 2 z}\right\rangle-\left\langle F_{i 2 z}\right\rangle\right)$. Thus, the electrons can accelerate, if the ions are mobile. If one considers the ions to be immobile (for high-frequency electron waves), so then the electrons do not accelerate due to the ponderomotive force $\left\langle F_{e 2 z}\right\rangle$, because this force is compensated for by the action of the electric field $\left\langle E_{2 z}\right\rangle$.

One of the most direct ways to investigate the dust grain dynamics under the action of the high-frequency radiation are active experiments in the Earth's mesosphere (Havnes et al., 2001). Parameters of radar beams, such as the power, duration, inhomogeneity, can be controlled. The displacement of dust layers under the radar beam action can be detected via the Polar Mesospheric Summer Echo.

The stationary ponderomotive force of Alfvén waves is popular at the explanation of the ion acceleration along the Earth's geomagnetic field (see, e.g. Allan and Manuel, 1996; Guglielmi and Lundin, 2001, and references therein). However, such an approach is not suitable for sufficiently short wave packets, the size of which is less than the inhomogeneity length of medium. In this case it is necessary to use the nonstationary ponderomotive force considered in the present paper.

It is possible that the temporal evolution of Alfvén waves can contribute to the interpretation of the creation of magnetic holes and magnetic decreases observed in the interplanetary magnetic field by means of the ponderomotive force (Tsurutani et al., 2002).

\section{Conclusions}

In this paper we have developed a general theory for the generation of nonlinear, low-frequency, electromagnetic fields, induced by the ponderomotive force of high-frequency radiations in cold, uniform, multicomponent, dusty magnetoplasmas. The high-frequency electromagnetic radiations have been considered to be both nonuniform in space and nonstationary in time. Taking into account the high-frequency wave amplitude nonstationarity is important in the consideration of the plasma particle dynamics due to the ponderomotive force. We have shown that depending on the character of the spatio-temporal inhomogeneity, the nonlinear electromagnetic fields induced by the ponderomotive force can change their direction, leading to the corresponding change in the particle velocity direction.

As a concrete example for high-frequency waves the electromagnetic cyclotron waves travelling along the background magnetic field have been considered. We have studied in detail the dynamics of dust grains under the action of the non- 
linear electromagnetic field induced by the cyclotron waves. Dust grains were considered to be magnetized and unmagnetized. We have shown that when the wave amplitude depends on time, different regimes of dust dynamics are possible: stationary, dynamic and quasi-dynamic. We have presented the conditions for when these regimes exist, and the equations for dust grain velocities. We have found that when the amplitude of the high-frequency wave does not depend on time (the purely static regime), the induced electric field is electrostatic and determined only by the electron motion. In all other cases this field has an electromagnetic nature. The transverse acceleration of dust grains in dynamic regimes is proportional to the Alfvén velocity. For the case $n_{d 0} m_{d} \ll n_{i 0} m_{i}$ this acceleration is identical for magnetized and unmagnetized grains, and proportional to the ion Alfvén velocity. However, when $n_{d 0} m_{d} \gg n_{i 0} m_{i}$ the acceleration of unmagnetized dust grains in the dynamic regime has the maximal magnitude, since it stays proportional to the ion Alfvén velocity, and the acceleration of the magnetized grains is proportional to the total Alfvén velocity determined by the dust grains in this case. For the latter case the quasi-dynamic regime for the unmagnetized dust grains is also possible, the transverse acceleration of which is the same as for magnetized grains in the dynamic regime. The quasi-dynamic regime has been considered in Shukla et al. (2002). However, the conditions for applicability of the results obtained are not given. In addition, the equations for the longitudinal acceleration of dust grains (or the expression for the nonlinear electric field along the background magnetic field) are erroneous in this paper.

We have not taken into account here the collisions of particles with neutral background. For transverse dust grain movements it is justified if $\max \left(\omega, \omega_{c d}\right) \gg v_{d n}$, where $v_{d n}$ is the collision frequency of dust grains with neutrals. For the longitudinal acceleration the collisions of dust grains are not essential on time scales of less than $\nu_{d n}^{-1}$, if $k_{z}\left(\omega^{\prime} / k_{z}^{\prime}\right) \gg \omega$. In the opposite case the condition $\omega \gg v_{d n}$ must be fulfilled.

The relationships between the transverse and longitudinal accelerations depend on the concrete regimes, high frequencies and plasma parameters. Knowledge of dusty plasma and wave parameters in specific situations is important for determining the dust grain dynamics. Under consideration of the possible regimes for nonlinear wave packets, it is necessary to take into account their movement with the group velocity.

The electron and ion slow dynamics is determined by both the ponderomotive force and the induced nonlinear electric field.

In our paper we have shown that there is a large variety of conditions determining the dynamics of dust grains under the action of the nonstationary ponderomotive force. Any possibilities can be realized for real objects. Some estimations for the dust acceleration can be found in the literature (Shukla et al., 2002; Shukla and Stenflo, 2002; Shukla and Schlickeiser, 2003).

The results obtained in the present paper may be useful for understanding the possible mechanisms of dust grain dynam- ics in astrophysical, cosmic and laboratory plasmas, under the action of nonuniform and nonstationary electromagnetic waves.

Acknowledgements. F. Z. Feygin acknowledges the financial support by the Commission of the European Union (research grant INTAS), the Russian Fund for Basic Research, research grant 05-0564992, and Russian Academy of Sciences through the grant "Lowfrequency nonlinear geomagnetic wave structures as a response of the magnetosphere to the solar wind perturbations".

Topical Editor U. P. Hoppe thanks R. Rankin and another referee for their help in evaluating this paper.

\section{References}

Abe, H. and Kadoya, Y.: Particle simulation of radio frequency stabilization of the flute mode in a tandem mirror, II. Perpendicular antenna, Phys. Fluids, 31, 3035-3043, 1988.

Akhiezer, A. I., Akhiezer, I. A., Polovin, R. V., Sitenko, A. G., and Stepanov, K. N.: Elektrodinamika plazmy, Nauka, Moscow, 191, 1974.

Allan, W. and Manuel, J. R.: Ponderomotive effects in magnetospheric hydromagnetic waves, Ann. Geophys., 14, 893-905, 1996.

Altobelli, N., Kempf, S., Landgraf, M., Srama, R., Dikarev, V., Krüger, H., Moragas-Klostermeyer, G., and Grün, E.: Cassini between Venus and Earth: Detection of interstellar dust, J. Geophys. Res., 108(A10), 8032, doi:10.1029/2003JA009874, 2003.

Barkan, A., D’Angelo, N., and Merlino, R. L.: Charging of dust grains in a plasma, Phys. Rev. Lett., 73, 3093-3096, 1994.

Chu, J. and Lin, I.: Direct observation of Coulomb crystals and liquids in strongly coupled rf dusty plasmas, Phys. Rev. Lett., 72, 4009-4012, 1994.

Draine, B. T. and Lazarian, A.: Electric dipole radiation from spinning dust grains, Astrophys. J., 508, 157-179, 1998.

Falceta-Goncalves, D. and Jatenco-Pereira, V.: The effects of Alfvén waves and radiation pressure in dust winds of late-type stars, Astrophys. J., 576, 976-981, 2002.

Ferrara, A. and Dettmar, R. J.: Radio-emitting dust in the free electron layer of spiral galaxies: Testing the disk/hallo interface, Astrophys. J., 427, 155-159, 1994.

Fisch, N. J. and Boozer, A. H.: Creating an asymmetric plasma resistivity with waves, Phys. Rev. Lett., 45, 720-722, 1980

Fortov, V. E., Nefedov, A. P., Vaulina, O. S. et al.: Dusty plasma induced by solar radiation under microgravitational conditions: an experiment on board the Mir orbiting space station, J. Exp. Theor. Phys., 87, 1087-1097, 1998.

Fränz, M., Burgess, D., and Horbury, T. S.: Magnetic field depressions in the solar wind, J. Geophys. Res., 105(A6), 12725 12 732, doi:10.1029/2000JA900026, 2000.

Goertz, C. K.: Dusty plasmas in the solar system, Rev. Geophys., 27, 271, 1989.

Guglielmi, A. and Lundin, R.: Ponderomotive upward acceleration of ions by ion cyclotron and Alfvén waves over the polar region, J. Geophys. Res., 106(A7), 13 219, doi:10.1029/2000JA900066, 2001.

Havnes, O., Brattli, A., Aslaksen, T., Singer, W., Latteck, E., Blix, T., Thrane, E., and Trøim, J.: First common volume observations of layered plasma structures and polar mesospheric summer 
echoes by rocket and radar, Geophys. Res. Lett., 28, 1419-1422, 2001.

Havnes, O., Melands $\varnothing$, F., Hartquist, T., and Aslaksen., T.: Charged dust in the Earth's mesopause: Effects on radar backscatter, Phys. Scr., 45, 535-544, 1992.

Havnes, O., Trøim, J., Blix, T., Mortensen, W., Naesheim, L. I., Thrane, E., and Tønnesen, T.: First detection of charged dust particles in the Earth's mesosphere, J. Geophys. Res., 101(A5), 10 839-10 848, 1996.

Ikezi, H.: Coulomb solid of small particles in plasmas, Phys. Fluids, 29, 1764-1766, 1986.

Jha, P., Wadhwani, N., Upadhyaya, A. K., and Raj, G.: Selffocusing and channel-coupling effects on short laser pulses propagating in a plasma channel, Phys. Plasmas, 11, 3259-3263, 2004.

Lee, N. C. and Parks, G. K.: Ponderomotive force in a warm twofluid plasma, Phys. Fluids, 26, 724-729, 1983.

Li, X. L. and Temerin, M.: Ponderomotive effects on ion acceleration in the auroral zone, Geophys. Res. Lett., 20(1), 13-16, 1993.

Liu, M., Guo, H., Li, B., Liu, S., and Wu, G.: Effect of wakefield on asymmetric self-phase modulation of an intense laser pulse propagating in a plasma in resonant and non-resonant cases, Phys. Lett. A, 327, 192-197, 2004.

Myra, J. R. and D'Ippolito, D. A.: Poloidal force generation by applied radio frequency waves, Phys. Plasmas, 7, 3600-3609, 2000.

Nekrasov, A. K. and Feygin, F. Z.: Ponderomotive action of ULF pulsations in the magnetospheric plasma, Phys. Scr., 71, 310313,2005

Ofman, L. and Davila, J. M.: Nonlinear resonant absorption of Alfvén waves in three dimensions, scaling laws, and coronal heating [Alfvén], J. Geophys. Res., 100(A12), 23 427-23 442, 1995

Rao, N. N., Shukla, P. K., and Yu, M.: Dust-acoustic waves in dusty plasmas, Planet. Space Sci., 38, 543-546, 1990.

Shukla, P. K.: Waves in dusty plasmas, Proceedings of the Sixth Workshop on "The physics of dusty plasmas", edited by: Shukla, P. K., Mendis, D. A., and Chow, V. W., La Solla, California, USA, 22-25 March, 1995.

Shukla, P. K. and Schlickeiser, R.: Dust grain acceleration by the ponderomotive force of Alfvén waves in cosmic plasmas, Phys. Plasmas, 10, 1523-1525, 2003.

Shukla, P. K. and Stenflo, L.: Acceleration of dust particles by the ponderomotive force of lower-hybrid waves, Phys. Lett. A, 293 , 188-190, 2002.

Shukla, P. K., Mendis, D. A., and Chow, V. W. (Eds.):The physics of dusty plasmas, Proceedings of the Sixth Workshop, La Solla, California, USA, 22-25 March, 1995.

Shukla, P. K., Stenflo, L., Bingham, R., and Dendy, R. O.: Ponderomotive force acceleration of ions in the auroral region, J. Geophys. Res., 101(A12), 27 449-27 452, 1996.

Shukla, P. K., Stenflo, L., Mamun, A. A., Resendes, D. P., and Sorasio, G.: Acceleration of dust grains by means of electromagnetic cyclotron waves, J. Geophys. Res., 107(A10), 1293, doi:10.1029/2002JA009321, 2002.

Spitzer Jr., L.: Physical Processes in the Interstellar Medium, Wiley, New York, 1978.

Thomas, H., Morfill, G. E., Demmel, V., Goree, J., Feuerbacher, B. and Möhlmann, D.: Plasma crystal: Coulomb crystallization in a dusty plasma, Phys. Rev. Lett., 73, 652-655, 1994.

Tsurutani, B. T., Dasgupta, B., Galvan, C., Neugebauer, M., Lakhina, G. S., Arballo, J. K., Winterhalter, D., Goldstein, B. E., and Buti, B.: Phase-steepened Alfvén waves, proton perpendicular energization and the creation of magnetic holes and magnetic decreases: The ponderomotive force, Geophys. Res. Lett., 29(24), 2233, doi:10.1029/2002GL015652, 2002.

Verheest, F.: Waves in Dusty Space Plasmas, Kluwer Academic Publ., Dordrecht, 2000. 